\title{
Editorial
}

\section{Transportation Modeling and Management}

\author{
Xiaoning Zhang, ${ }^{1}$ Henk van Zuylen, ${ }^{2}$ Yafeng Yin, ${ }^{3}$ Andy H. F. Chow, ${ }^{4}$ and Hu Shao ${ }^{5}$ \\ ${ }^{1}$ School of Economics and Management, Tongji University, Shanghai 200092, China \\ ${ }^{2}$ Department of Transport and Planning, Delft University of Technology, Delft, The Netherlands \\ ${ }^{3}$ Department of Civil and Coastal Engineering, University of Florida, Gainesville, FL 32611, USA \\ ${ }^{4}$ Department of Civil, Environmental and Geomatic Engineering, University College London, London, UK \\ ${ }^{5}$ Department of Civil \& Structural Engineering, The Hong Kong Polytechnic University, Hong Kong \\ Correspondence should be addressed to Xiaoning Zhang; cexzhang@tongji.edu.cn
}

Received 19 October 2014; Accepted 19 October 2014; Published 24 December 2014

Copyright (C) 2014 Xiaoning Zhang et al. This is an open access article distributed under the Creative Commons Attribution License, which permits unrestricted use, distribution, and reproduction in any medium, provided the original work is properly cited.

Due to the population growth and the rapid progress of urbanization, most big cities over the world face serious problems of traffic congestion, traffic safety, and vehicular emission. It is an urgent need to develop new models and new methods to describe and optimize the transportation system. This special issue is devoted to publishing the latest and significant results on scientific research in the mathematical methods of modeling and managing transportation systems.

After conducting rigorous peer review, this special issue has accepted 28 technical papers focusing on transportation modeling and management. We describe each one of them concisely as follows.

In "A day-to-day route choice model based on reinforcement learning," $\mathrm{F}$. Wei et al. propose a day-to-day route choice model based on reinforcement learning and multiagent simulation. Travelers' memory, learning rate, and experience cognition are taken into account. Both the scenarios of link capacity degradation and random link capacity are used to illustrate the model's applications. Analyses and applications of the model demonstrate that the model is reasonable and useful for studying the day-to-day traffic dynamics.

In "A schedule optimization model on multirunway based on ant colony algorithm," Y. Jiang et al. develop a schedule optimization model for arrival-departure flights, to make full use of the slot of runway, reduce flight delay, and ensure fairness among airlines. The total delay cost and fairness among airlines are two objective functions. The ant colony algorithm is adopted to solve this problem and the result is more efficient and reasonable compared with FCFS (first come first served) strategy. Optimization results show that the flight delay and fair deviation are decreased by $42.22 \%$ and $38.64 \%$, respectively.

In "Grain emergency vehicle scheduling problem with time and demand uncertainty," J. DongQing and Z. QunXiong study the grain transportation in relief and emergency supply chains. They take the grain emergency vehicle scheduling model between multiwarehouses as the research object. Under the emergency environment, the aim is to maximize the utilization of vehicles and minimize the delay. The randomness of the key parameters in grain emergency vehicle scheduling is determined through statistical analysis and the model is solved through robust optimization method. It is shown that the uncertainty of both time and demand has great influence on the efficiency of grain emergency vehicle scheduling problem.

In "Analysis of time of day fare discounts on urban mass transit travel behavior, crowding, and waiting time," $X$. Guo and H. Sun develop models to find an optimal discount fare and time intervals on morning peak hour. Two models have been analyzed to describe it with schedule delay because of the travel demand size. The first objective function is constructed on pressure equalization when the travel demand is small. The other objective function is to minimize total waiting time when the travel demand is large. In the end, numerical examples based on an artificial network are performed to characterize fare discount models.

In "The bilevel design problem for communication networks on trains: model, algorithm, and verification," Y. Tian et 
al. propose a novel method to solve the problem of train communication network design. Firstly, they put forward a general description of such problem. Then, taking advantage of the bilevel programming theory, they create the costreliability-delay model (CRD model). They use a practical example to verify the accuracy and the effectiveness of the CRD model and further apply the novel method on a train with six carriages.

In "Managing rush hour congestion with lane reversal and tradable credits," Q. Li and Z. Gao develop models to reallocate the two-way road lane to cater for the asymmetric traffic flow. On the other hand, an effective tradable credit scheme is proposed to reduce the traffic demand and improve fairness for all travelers. A discrete bilevel programming model is established. The government at the upper level reallocates lanes on the two-way road to minimize the total system cost. The travelers at the lower level choose the optimal route on the basis of both travel time and credit charging for the lanes involved.

In "Model of wagons' placing-in and taking-out problem in a railway station and its heuristic algorithm," C. Guo and D. Lei develop models to decrease wagons' dwell time in railway stations and improve the efficiency of railway transportation. They take the locomotive running times between goods operation sites as weights, so the problem is described by a single machine scheduling problem, which is solved by a Hungarian algorithm.

In "Developing a collaborative planning framework for sustainable transportation," O. Ö. Özener considers a delivery network with multiple customers served by a single carrier. It executes a delivery plan with the minimum transportation cost and allocates the resulting costs and the emissions among the customers in a fair manner. In order to develop a mechanism that provides further reduction of the emissions, he studies a setting where the carrier takes the responsibility of the emissions and reflects the resulting inefficiencies while charging the customers.

In "Storage space allocation of inbound container in railway container terminal," L. Wang et al. consider the formulation and solution algorithm for storage space allocation problem of inbound containers in railway container terminal. The problem is formulated as two-stage optimization models, whose objectives are balancing the workload of inbound containers and reducing the overlapping amounts. An algorithm implement process based on rolling-horizon approach is designed to solve the proposed models. Real application shows that the proposed approach is effective to solve space allocation problem of inbound container and is significant for the operation and organization of railway container terminals.

In "A bayesian combined model for time-dependent turning movement proportions estimation at intersections," P. Jiao et al. present a back propagation neural network model to estimate dynamic turning movements, as well as the selfadaptive learning rate approach and the gradient descent with momentum method for solving. They design a revised sequential Kalman filtering algorithm. A field survey is implemented at an intersection in Beijing city to collect both time series of link counts and actual time-dependent turning movement flows, including historical and present data. The reported estimation results show that the Bayesian combined model is much more accurate and stable than other models.

In "Multiagent based decentralized traffic light control for large urban transportation system," Y. Xu et al. propose a novel decentralized multiagent based approach for massive traffic lights coordination to promote the large-scale green transportation. Within a local intersection, constraint optimizing agents are designed to efficiently search for joint activities of the lights.

In "Modeling complex system correlation using detrended cross-correlation coefficient," K. Dong et al. take traffic dynamic as an example of a complex system. By applying the detrended cross-correlation coefficient method to traffic time series, they find that the traffic fluctuation time series may exhibit cross-correlation characteristic. Further, they show that two traffic speed time series derived from adjacent sections exhibit much stronger cross-correlations than the two speed series derived from adjacent lanes. Similarly, they also demonstrate that the cross-correlation property between the traffic volume variables from two adjacent sections is stronger than the cross-correlation property between the volume variables of adjacent lanes.

In "A two-stage model for project optimization in transportation infrastructure management system," Z. Chen et al. propose a novel two-stage project optimization model, including budget allocation and project distribution. Moreover, the methods of dynamic programming (DP) and genetic algorithm (GA) are applied to obtain an effective solution. The findings indicate that the new optimization method can provide a satisfactory and reasonable maintenance schedule for transportation infrastructure maintenance agencies whose routine management will benefit from the newly proposed model.

In "Exploring urban taxi drivers' activity distribution based on GPS data," X. Hu et al. analyze the urban taxi driver's activity distribution characteristics from different temporal and spatial levels. In the time level, they identify the difference with taxi daily operation pattern (weekday versus weekends), continuous time in one day, passengers in vehicle time, and taxi drivers' operation frequency; in the space level, they explore the taxi driver's searching pattern, including searching activity space distribution and the relationship between the pick-up locations and the drop-off locations. It is helpful for urban taxi drivers' operation and behavior pattern identification, as well as the contribution to the geographical activity space analysis.

In "Measurement of international roughness index by using Z-axis accelerometers and GPS," Y. Du et al. develop a measurement system equipped with $Z$-axis accelerometers and a GPS device. Using the self-designing measurement system based on the methodology proposed in this study, they perform a small-scale field test. They use a one-wheel linear model and two-wheel model to fit the variation of the $Z$-axis acceleration. The test results demonstrate that the low-cost measurement system has good accuracy and could enhance the efficiency of IRI measurement.

In "Uncertain programming for network revenue management," D. Mou and X. Wang present a chance-constrained 
programming model based on the uncertainty theory for network revenue management, in which the fares and the demands both are uncertain variables rather than random variables. Based on the strategy of nested booking limits, a solution method of booking control is developed to solve the problem.

In "Optimal coordinated strategy analysis for the procurement logistics of a steel group," L. Deng et al. study the optimization of an internal coordinated procurement logistics system in a steel group and the decision on the coordinated procurement strategy by minimizing the logistics costs. Considering the coordinated procurement strategy and the procurement logistics costs, the aim of the optimization model was to maximize the degree of quality satisfaction and to minimize the procurement logistics costs.

In "An integrative approach with sequential game to realtime gate assignment under CDM mechanism," J.-q. Liu et al. study real-time airport gate assignment problem when smallscale or medium- to large-scale flight delays occur. Taking into account the collaborative decision making (CDM) of the airlines and the airport, slot assignment and gate assignment are integrated into mixed set programming (MSP), and a realtime gate assignment model is built and solved through MSP coupled with sequential game.

In "Energy and environmental efficiency of China's transportation sector: a multidirectional analysis approach," G. Bi present a nonradial DEA model with multidirectional efficiency analysis (MEA) involving undesirable outputs for the measurement of regional energy and environmental efficiency of China's transportation sector during the period 2006-2010. They not only evaluate the energy and environmental efficiency level and trend of China's transportation sector but also investigate the efficiency patterns of 30 regions and three major areas of China.

In "Synthetic optimization model and algorithm for railway freight center station location and wagon flow organization problem," X.-c. Liu et al. propose a two-stage method to optimize railway freight center stations location and wagon flow organization together. The location model is present with the objective to minimize the operation cost and fixed construction cost. Then, the second model of wagon flow organization is proposed to decide the optimal train service between different freight center stations. A heuristic algorithm that combined tabu search (TS) with adaptive clonal selection algorithm (ACSA) is proposed to solve those two models.

In 'SIRS model of passengers' panic propagation under self-organization circumstance in the subway emergency," $\mathrm{H}$. Zhao et al. develop an improved SIRS model of passengers' panic spread in subway emergency with consideration of passengers' density, the characteristic of subway car with the confined space, and passengers' psychological factors. It helps the government and subway administration to master the panic spread mechanism and reduce the panic spread by improving measures and also provides certain reference significance for rail system construction, emergency contingency plans, and the construction and implementation of emergency response system.
In "Berth allocation problem with quay crane assignment for container terminals based on rolling-horizon strategy," L. Xiao and Z.-H. Hu develop a multiobjective optimization model which minimizes the total penalty costs. Then, the scheduling process is divided into a set of continual scheduling interval according to the dynamic arrival sequences. Meanwhile, rolling-horizon strategies for setting rolling and frozen windows and the parameter updating strategy are designed. The input parameters of the model in the next rolling window are updated according to the optimal results of each time window which have been obtained.

In "Day-to-day scheduling travel time adjustment behavior and simulation," H.-M. Li et al. propose a modeling framework to study the day-to-day scheduling travel time adjustment behavior on the basis of past experiences. Scheduling travel time is defined as the difference between the requested arrival time and the departure time. Mathematical equations are established to formulate every traveler's dynamic adjustment on his/her departure time.

In "Traffic incident clearance time and arrival time prediction based on hazard models," Y. b. Ji et al. develop some hazard based prediction models for both incident clearance time and arrival time. The best fitting distributions are drawn for both clearance and arrival time for 3 types of incident: crash, stationary vehicle, and hazard. The results show that Gamma, Log-logistic, and Weibull are the best fit for crash, stationary vehicle, and hazard incident, respectively. The obvious impact factors are given for crash clearance time and arrival time. The quantitative influences for crash and hazard incident are presented for both clearance and arrival. The model accuracy is analyzed at the end.

In "Transportation network design considering morning and evening peak-hour demands," $\mathrm{H}$. Wang et al. propose an NDP model simultaneously considering both morning and evening peak-hour demands. The NDP problem is formulated as a bilevel programming model, where the upper level is to minimize the weighted sum of total travel time for network users travelling in both morning and evening commute peaks, and the lower level is to characterize user equilibrium choice behaviors of the travelers in two peaks. The proposed NDP model is transformed into an equivalent mixed integer linear programming (MILP), which can be efficiently solved by optimization solvers.

In "Trafficability analysis at traffic crossing and parameters optimization based on particle swarm optimization method," B. He and Q. Lu propose a mathematical model for the platoon's longitudinal movement. A systematic analysis of longitudinal control law is presented for the platoon of vehicles. The particle swarm optimization method is introduced to effectively optimize the parameters of platoon. The proposed method effectively finds the optimal parameters based on simulations and makes the spacing of platoon shorter.

In "Investigating the in-vehicle crowding cost functions for public transit modes," F. Qin conducts both quantitative and qualitative studies, focusing on remodeling the invehicle crowding cost functions for different transit modes. Three numerical case studies show that applying distinct invehicle crowding cost functions to different transit modes has implications not only for the cost structure of transit systems 
and the magnitude of optimal service provisions but also for the presence of economies of scale in consumption.

In "Potential field cellular automata model for pedestrian evacuation in a domain with a ramp," X.-X. Jian and X. Zhang propose a potential field cellular automata model with a pushing force field to simulate the pedestrian evacuation in a domain with a ramp. They construct a cost potential depending on the ramp angle and introduce a function to evaluate the pushing force, which is related to the cost and the desired direction of pedestrian. The relationship between the slope of ramp and the pushing force is investigated. The changing of injured situations with the changing of the slope of ramp is also studied. When the number of pedestrians and the ramp angle arrive at certain critical points, the domino effect is simulated by this proposed model.

We think that the special issue presents many innovative and meaningful studies on transportation modeling and management related problems. We hope the published papers will provide a good foundation for future scientific research related to transportation management and optimization.

\section{Acknowledgments}

We also express our hearty thanks to all the contributors for sharing their excellent research works to this special issue and the anonymous reviewers for their time and effort in providing constructive and helpful reviews.

Xiaoning Zhang

Henk van Zuylen Yafeng Yin

Andy H. F. Chow

$\mathrm{Hu}$ Shao 


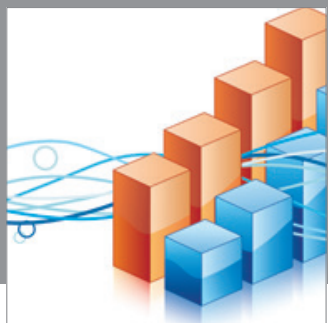

Advances in

Operations Research

mansans

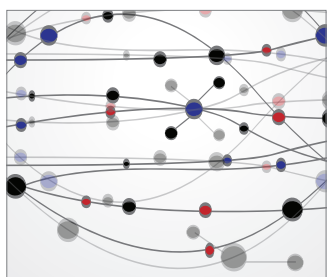

The Scientific World Journal
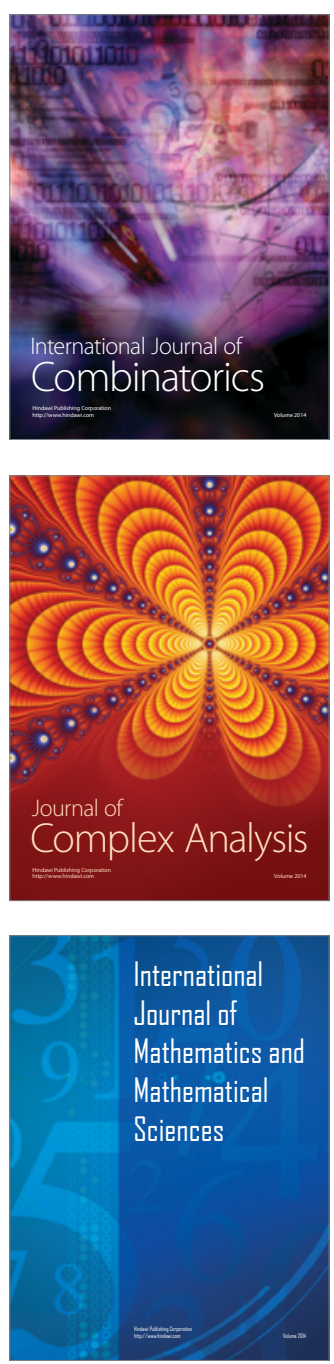
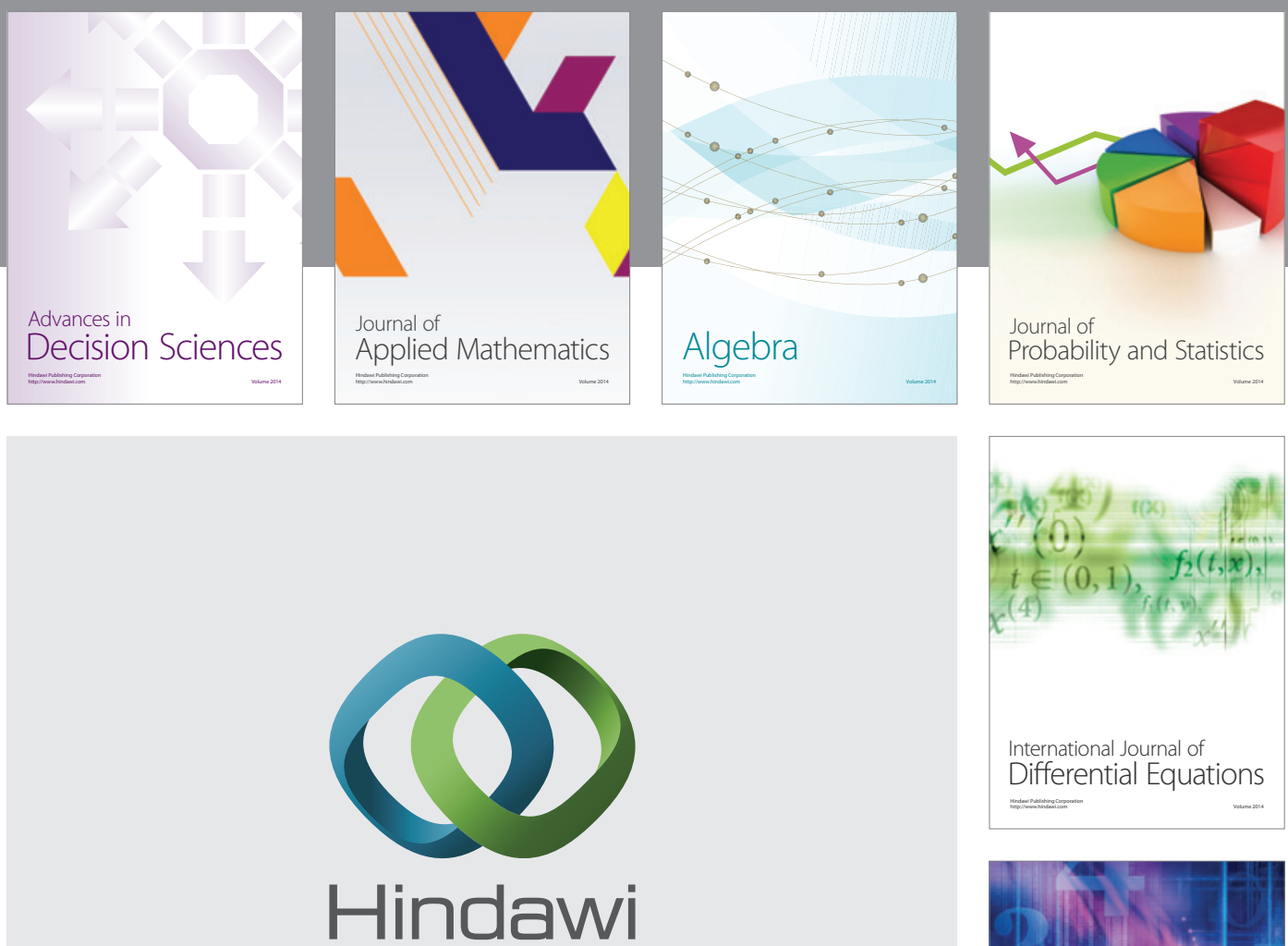

Submit your manuscripts at http://www.hindawi.com
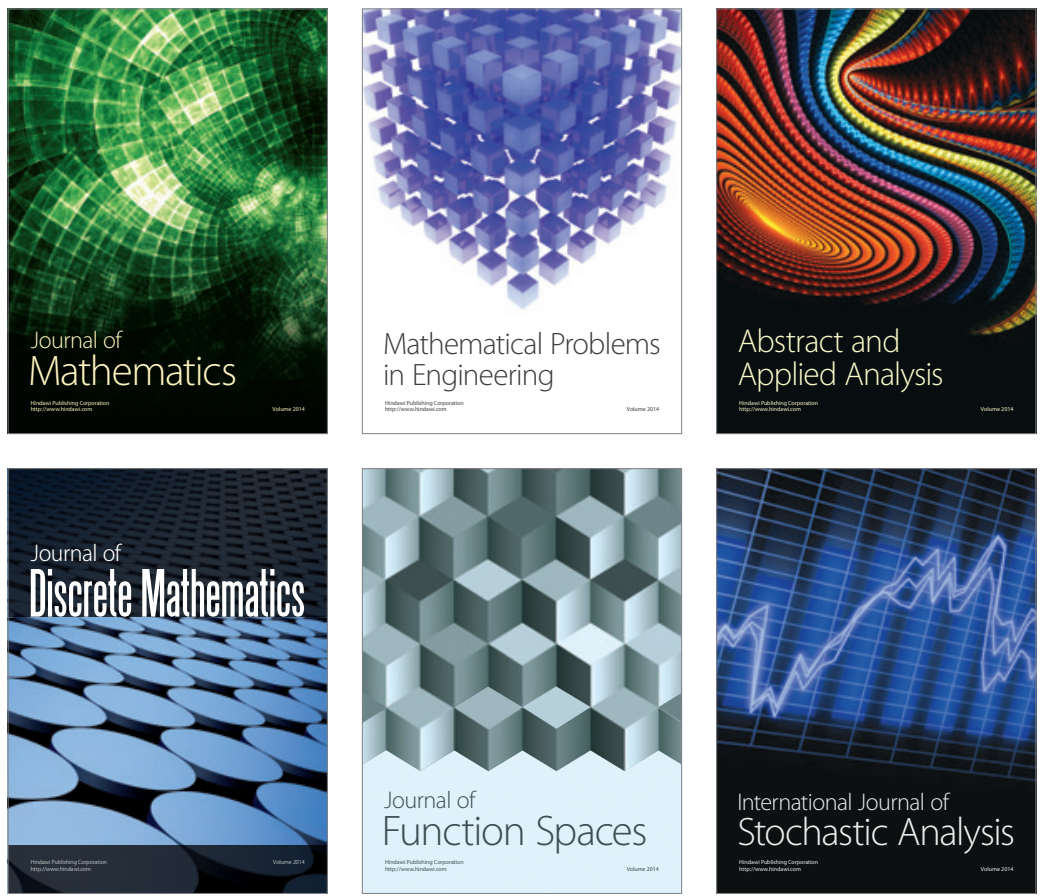

Journal of

Function Spaces

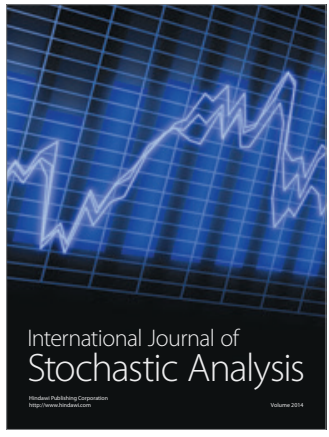

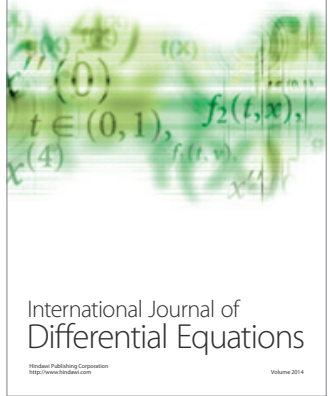
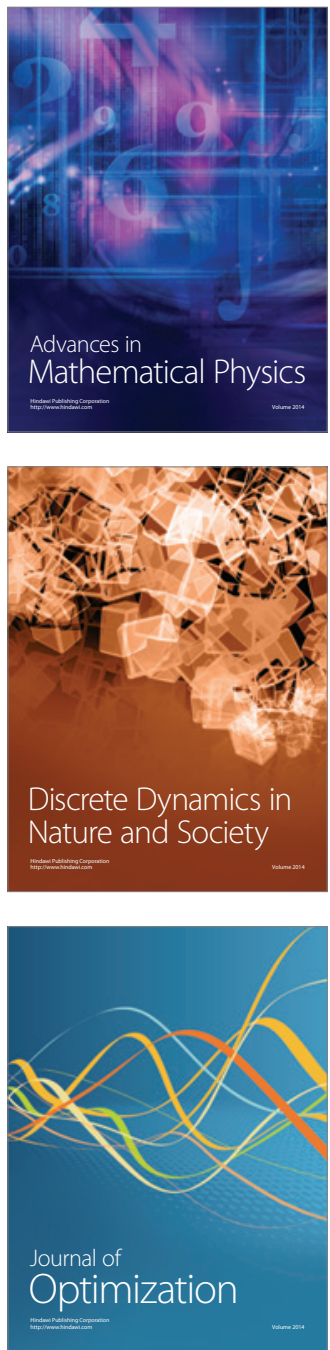